\title{
Empirical Studies of the Value of Conceptually Explicit Notations in Collaborative Learning
}

\author{
Daniel D. Suthers ${ }^{1}$ \\ 1 University of Hawai i at Manoa, Department of Information and Computer Sciences, \\ suthers@hawaii.edu
}

\begin{abstract}
Knowledge Cartography" is concerned with a diversity of notations that all make certain conceptual structures explicit, but may differ from each other and from conceptually implicit notations in what they make salient. This chapter reports on a series of studies that investigated the idea that these differences or representational biases might lead to differences in processes of collaborative inquiry. The studies span face-to-face, synchronous online and asynchronous online media in both classroom and laboratory settings. An understanding of the observed effects can help both designers and practitioners think more deeply about the pedagogical implications of their representational tools and how these tools are embedded in a learning situation; i.e., how to convert representational biases to representational guidance.
\end{abstract}

\section{Introduction}

The variety of representational tools discussed in this volume-argument maps, concept maps, evidence maps, knowledge maps, mind maps, etc.- -all offer the common advantage of being explicit about some conceptual structure or model: their notations are for constructing conceptually explicit representational artifacts. (See Suthers, 2001b for discussion of the distinction between notation, tool and artifact.) In contrast written language is far more expressive yet as a notation does not make any particular conceptual structure visually salient. Researchers have claimed that explicit representations of conceptual structure encourage participants to clarify their thinking (Brna, Cox, \& Good, 2001), make this thinking visible to others (Bell, 1997), provide resources for conversation (Roschelle, 1996), can guide students' argumentation to include disconfirming as well as confirming evidence (Toth, Suthers, \& Lesgold, 2002; Veerman, 2003), and can function as a "convergence artifact" that expresses the group's emerging consensus (Hewitt, 2001; Suthers, 2001a). The 
present chapter summarizes a series of studies undertaken to test hypothesized advantages of conceptually explicit notations, and that led to further discovery and explorations in the roles of representational tools in mediating interaction. The chapter begins with the historical context and motivation for the work and some theoretical considerations that led to the studies. The bulk of the chapter summarizes a series of classroom and laboratory studies comparing evidence maps to other representational notations, before concluding with some implications for practitioners.

\section{Background}

This section summarizes the practical and theoretical motivations for the studies that will be described in the next section.

\subsection{Belvedere and Kin}

This line of work had its origins in the Belvedere project at the University of Pittsburgh. The project was intended to support secondary school children's learning of critical inquiry skills in the context of science, particularly at the scale of scientific discourse that spans multiple studies and authors (Cavalli-Sforza, Weiner, \& Lesgold, 1994). Belvedere was intended to enable the construction of node-and-link style diagrams using a complex visual language that could capture the nuances of scientific argumentation, and an intelligent tutoring system that would help the student reason about the arguments. The name "Belvedere" was chosen by Alan Lesgold to convey both the "beautiful views" of arguments that it would enable, and the guidance it offered children like the butler "Mr. Belvedere" in a locally set television show. ${ }^{1}$

A prototype that included a portion of the visual language and a simple pattern matching advisor was implemented (Paolucci, Suthers, \& Weiner, 1996; Suthers \& Weiner, 1995; Suthers, Weiner, Connelly, \& Paolucci, 1995). Belvedere's diagrammatic language was later simplified in version 2 (Figure 1) to focus on evidential relations between data and hypotheses (Suthers et al., 2001). ${ }^{2}$ This change was driven in part by a refocus on collaborative learning, which led to a reconceptualization of the role of the diagrammatic representations. When more than one student was working with Bevledere, much of students' argumentation took place verbally between them rather than in the representations, and was concerned with manipula-

${ }^{1}$ Personal communication, Alan Lesgold.

${ }^{2}$ Version 4 of Belvedere is available at http://belvedere.sourceforge.net/. It supports multiple views on an evidence model, but does not support networked collaboration or include the prototype coach found in version 2. Version 2 is available from the author, but is based on 1990's technology. 


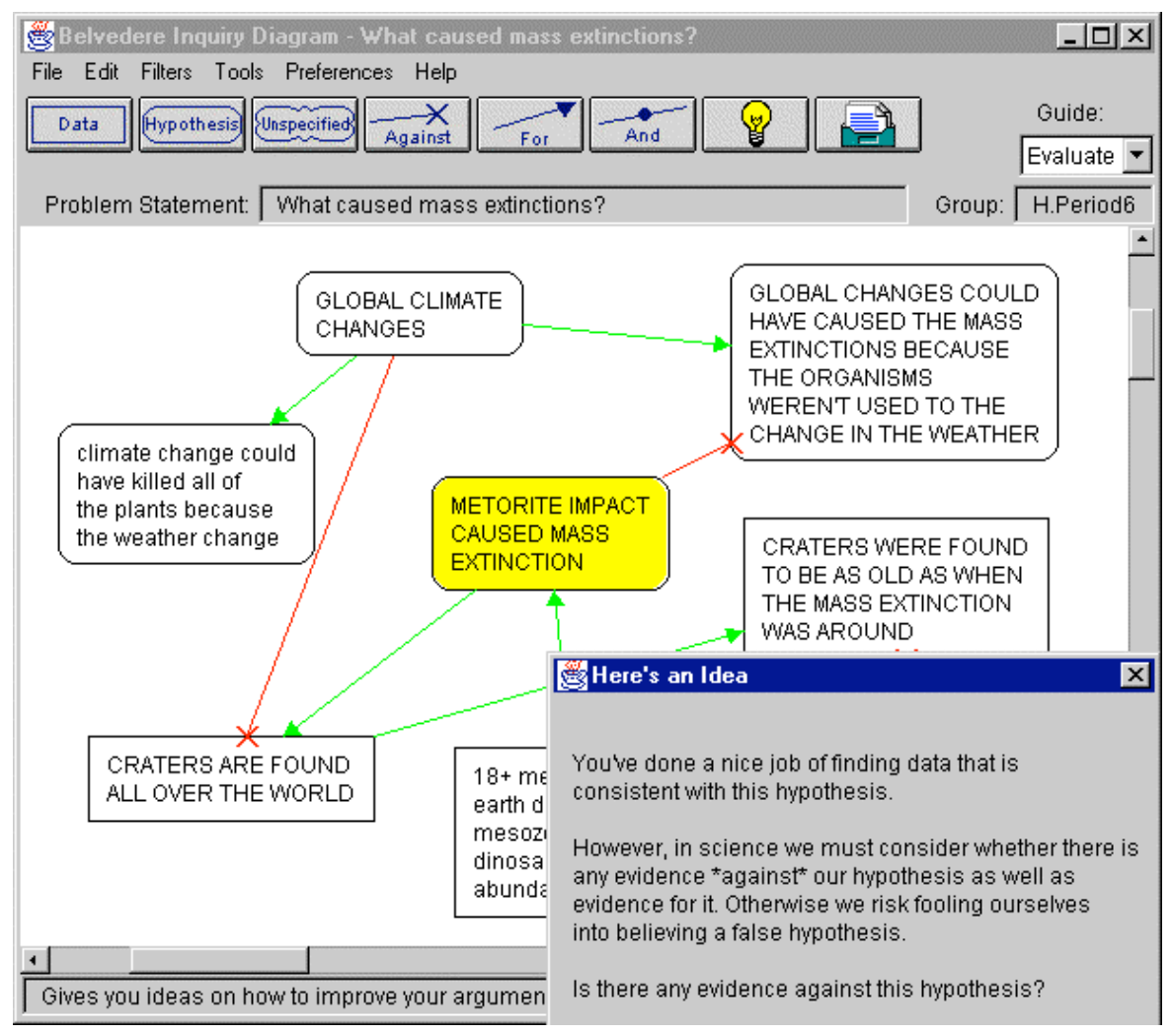

Figure 1. Belvedere 2, with prototype coach

tions and interpretations of the representations. Rather than viewing the representations as medium of communication or a formal record of an argumentation process, the author came to view them as resources (stimuli and guides) for conversation (Roschelle, 1996) among co-located learners concerning issues of evidence.

Meanwhile, it was apparent that various projects with similar goals (i.e., critical inquiry in a collaborative learning context) were using radically different representational systems. These included various forms of hypertext/hypermedia (Guzdial et al., 1997; O'Neill \& Gomez, 1994; Scardamalia et al., 1992), node-link graphs representing rhetorical, logical, or evidential relationships between assertions (Ranney, Schank, \& Diehl, 1995; Smolensky, Fox, King, \& Lewis, 1987; Suthers \& Weiner, 1995), containment of evidence within theory boxes (Bell, 1997), and evidence or criteria matrices (Puntambekar, Nagel, Hübscher, Guzdial, \& Kolodner, 1997). The obvious question arose: if representations are resources for conversation, does it matter which representation one uses? 


\subsection{Theoretical Background}

In response to this question, the author postulated two broad ways in which representational notations influence learning (Suthers, 2001b):

Constraints: limits on expressiveness, for example, the representational system may provide limited types of objects and relations and structures that can be constructed from them (Stenning \& Oberlander, 1995)

Salience: how the notation makes certain types of information (such as conceptual relationships) visible, possibly at the expense of others (Larkin \& Simon, 1987; Lohse, 1997). The absence of information where it is expected is also a form of salience (e.g., the empty cells of a matrix suggest that they might be filled).

These two fundamental expressive features of notations play out in many ways, including influences on individual (cognitive/perceptual) reasoning and learning (e.g., Kotovsky \& Simon, 1990; Novick \& Hmelo, 1994; Zhang, 1997), but here we are concerned with collaborative learning. Of the various influences that representations have on collaborative processes, which are intrinsic to collaborative processes themselves rather than being due to the aggregated influence of representations on individuals? Three possible answers to this question, first outlined in (Suthers \& Hundhausen, 2003) and further developed in (Suthers, 2006b), motivated the work reported in this chapter:

Negotiation Potentials. If multiple participants can add to or change a representational artifact that they are constructing together, the participants may feel an obligation to negotiate and obtain agreement on modifications to those representations. Any medium offers certain potentials for action ("affordances"). The ideas associated with these potential actions are more likely to be discussed in the course of this negotiation. Notational constraints limit but focus these negotiation potentials, while salience makes them more likely to be taken up by participants.

Referential Resource. When people are constructing representations together, elements of the representational artifact become imbued with meanings for the participants by virtue of having been produced through the process of negotiation discussed above. These elements then enable participants to reinvoke these meanings through language, gesture, or direct manipulation. In this manner, collaboratively constructed external representations facilitate subsequent negotiations, increasing elaboration on previous conceptions and the conceptual complexity that can be handled in group interactions. Constraints on expressiveness will focus what is available for reference, and salience will affect the immediacy of its availability for reference.

Mutual Awareness. Computational media can be designed to foster group awareness (Kreijns \& Kirschner, 2004). The mere awareness that others are present and will evaluate one's actions may influence one's choice of actions (Erickson \& Kellogg, 2000). An individual working in a group must constantly refer back to the shared external representation while coordinating activities with others: in- 
formation about the attentional status of group members and their attitudes towards previously proposed ideas may influence the actions of individuals in the group.

Following this reasoning, the author constructed a taxonomy of the various representations in use by researchers at the time, and made predictions such as the following:

- A plain text environment (e.g., a word processor) does not constrain expressiveness in any particular way (written language is very expressive), but nor does it make any particular relationships salient (e.g., one cannot tell "at a glance" the overall argumentative, conceptual, or evidential structure of a text).

- A graphical (node-link) tool such as Belvedere (e.g., Figure 1) will prompt users to make connections: all new contributions will be related to something else. Since participants talk about what they will do, this means, for example, that users of an evidence map are more likely to talk about evidence (as well as represent it) when using a graphical representation than plain text. Statements and the evidential relationships between them will be visually salient, so are more likely to be referenced in subsequent discussion, again leading to more talk about evidence.

- The salience of all the empty cells of a matrix (tabular) representation (e.g., to be shown in Figure 2) will prompt users to consider many possible relationships that can be expressed in those cells. For example, if hypotheses label the columns and data label the rows, users are more likely to talk about evidential relationships between the two, even more so than with a graph representation.

Predictions were made for other representational notations as well, but due to limited resources and the desire to sample diverse points in the design space of notations, the research to be discussed below was undertaken with these three notations. It should be understood that the research was not concerned with demonstrating the efficacy of these specific notations for learning. Rather, it sought to evaluate the idea that representations influence interaction in predictable ways that can be leveraged to influence the quality of collaborative learning. That is, we sought to show that representational bias exists (i.e., notational differences influence collaborative processes), which can be leveraged for representational guidance of learning.

\section{A Summary of the Research}

A series of studies were undertaken with various versions of software derived from Belvedere to test the effects of selected representations on collaborative inquiry. These studies include a classroom study and laboratory studies. The classroom study provided evidence that representational bias influences students' work in classroom settings. The laboratory studies provided a closer look at the effects of representa- 
tional bias on learning processes under controlled conditions, with a particular focus on the predictions just stated. Subsequently we shifted our focus to online settings.

\subsection{Guidance for Inquiry in a Classroom Setting}

Eva Toth, Arlene Weiner and the author developed a comprehensive method for implementing Belvedere-supported collaborative inquiry in the classroom (Suthers, Toth, \& Weiner, 1997; Toth et al., 2002). Students work in teams to investigate "science challenge problems" that present a phenomenon to be explained (e.g., the Cretaceous mass extinctions; the cause of a disease on the island of Guam), along with indices to relevant resources. The teams plan their investigation, perform hands-on experiments, analyze their results, and report their conclusions to others. Investigator roles are rotated between hands-on experiments, tabletop data analysis, computerbased literature review, and use of modeling tools such as Belvedere (we used the version of Figure 1). Assessment rubrics are given to the students at the beginning of their project as criteria to guide their activities. The rubrics guide peer review, and help the teacher assess learning objectives pertaining to inquiry in science. For further information on this integrated approach to classroom implementation, see (Suthers et al., 1997; Toth et al., 2002). ${ }^{3}$

As part of this work, we conducted a classroom study comparing two forms of guidance for inquiry with respect to quality of inquiry process and conclusions (Toth et al., 2002). The forms of guidance included Belvedere's graphical representations of evidential relations, and assessment rubrics. The Belvedere graphs relate data and hypothesis objects (represented by distinct shapes) with consistency and inconsistency relations (represented by links labeled "+" and "-"). The assessment rubrics were paper-based charts that included detailed criteria for progress in data collection, evaluation of information collected, quality of reports, and quality of peer presentations. Criteria used in the rubrics included the following:

- "The teams' work is composed of information found in multiple sources."

- "The content of the information the team used is related to the question asked."

- "The team considered multiple hypotheses that are appropriate to explain the scientific problem in question."

- "The team lists data for each hypothesis they have."

- "The team lists data against each hypothesis they have."

- "The team's work includes a conclusion summarizing the results of inquiry from various sources."

- "The report describes how the artifacts of investigations were used to analyze data and to formulate explanations and draw conclusions."

- "The presentation was clear, well organized and easy to follow."

${ }^{3}$ Supporting materials, including science challenge problems and assessment rubrics, are archived at http://lilt.ics.hawaii.edu/belvedere/index.html. 
The rubrics were provided to students at the outset of the study with explicit instructions to use them during the activity to guide inquiry. A $2 \times 2$ design crossed Belvedere versus Microsoft Word ${ }^{T M}$ conditions with Rubric versus No-Rubric conditions across four 9th grade science classes in U.S. Department of Defense Dependent Schools in Würzburg, Germany. Students spent about 2 weeks on each of three science challenge problems.

The data analysis was based primarily on artifacts produced by groups of students, namely their Belvedere graphs or Word documents, and their final report essays. The amount of information recorded did not differ significantly between groups. Significant results were obtained on the categorization of information and the number of evidential relationships recorded. An interaction between the type of representational tool and the use of rubrics prompted a post-hoc comparison. We found that the combination of graphing and rubrics resulted in a larger number of evidential relations recorded compared to all other conditions. Further analysis showed that this interaction was primarily due to the Belvedere/Rubrics students having recorded significantly more inconsistency relations. Thus, there appears to be a synergistic effect between effective representations and guidelines for their use, particularly with respect to attending to discrepant evidence. The best results were obtained with the combination of rubrics encouraging students to look for and record disconfirming as well as confirming information and explicit representational devices for recording such inferences. This result is consistent with other work on "distributed scaffolding" (Tabak, 2004). These results suggest that representational tools be designed together with other instructional interventions.

\subsection{Comparing Three Representations in a Laboratory Setting}

Subsequent laboratory studies were undertaken to document representational guidance in a controlled setting and to observe processes of representational guidance (we were not present during the classroom implementation in Germany). With the assistance of Christopher Hundhausen and Laura Girardeau, the author conducted a study comparing three alternative notations for recording evidential relationships between data and hypotheses with respect to participants' amount of talk about evidential relations (Suthers \& Hundhausen, 2003). We employed a single-factor, between-subjects design with three participant groups defined by the notation they used. Participants in the control group, Text, were given a simple word processor offering control over font characteristics and basic formatting. Participants in the Matrix condition used a tabular representation in which hypotheses were recorded as column headers, data were recorded as row headers, and each cell provided a menu for selecting symbols ("+," "-," "?," or a blank space) to indicate the relationship between the data item labeling the row and the hypothesis labeling the column (Figure 2). Participants in the Graph condition used a Belvedere-like evidencemapping tool (similar to Figure 3, but without the chat). Dependent measures included: (a) categorization of utterances and participant actions in the software; (b) ability to recall the data, hypotheses, and evidential relations explored in a multiple- 


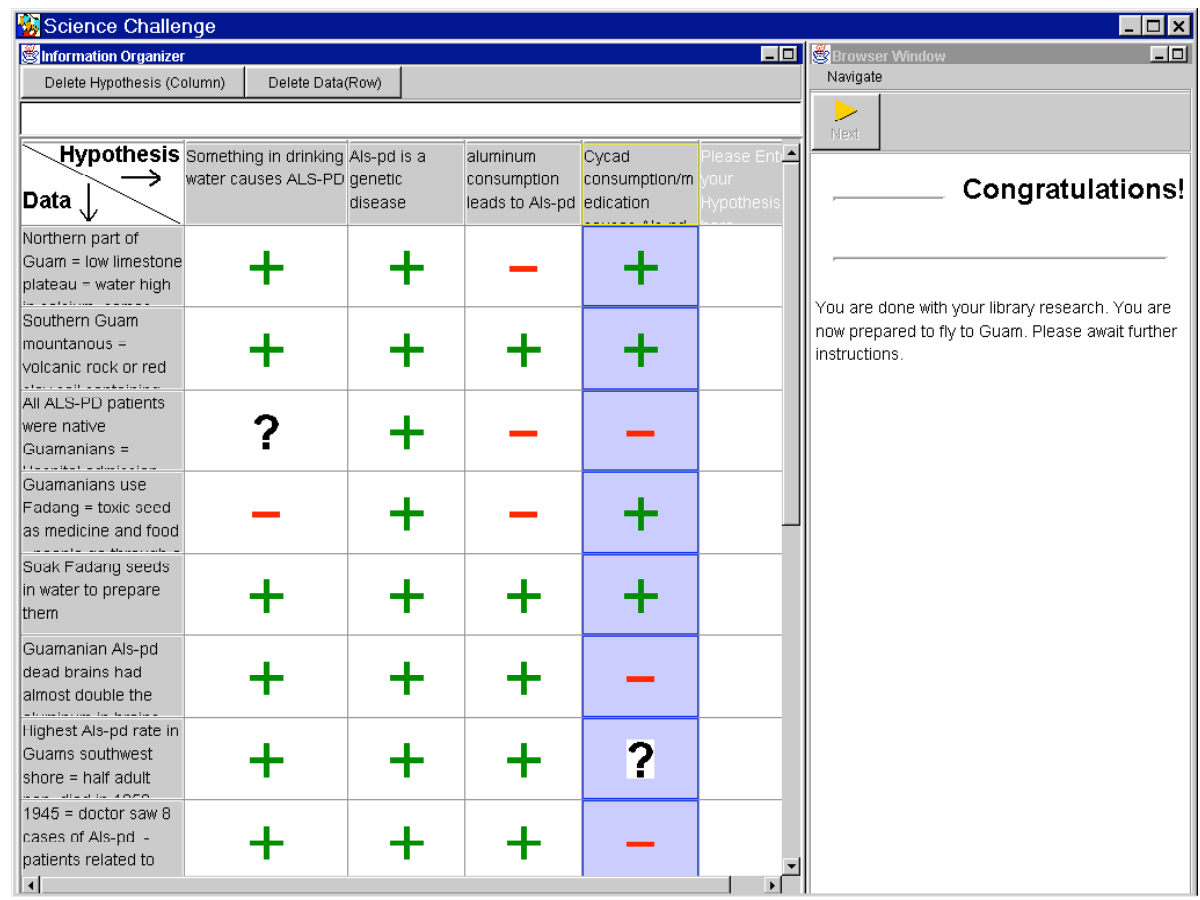

Figure 2. "Matrix" software, face-to-face study

choice test; and (c) ability to identify, in a written essay, the important evidential relations between the data and hypotheses presented.

Sixty students (in addition to students for a pilot study) were recruited out of introductory undergraduate science courses in same-gender pairs of self-selected acquaintances and randomly assigned to the three treatment groups under the constraint that the treatment groups were gender balanced with respect to Female/Female, Female/Male and Male/Male pairs. The experimental software had two main windows, one containing a workspace for creating either text, graph, or matrix representations, and the other presenting a science problem (e.g., to identify the cause mass extinctions, or of a neurological disease on the island of Guam) as a fixed sequence of 15 information pages available to both participants. Participants were instructed to visit each page in the sequence, and to record data, hypotheses, and evidential relations in their workspace. Once finished, they were individually given a post-test, and then asked to work together on an essay summarizing their findings.

All 30 sessions were videotaped and transcribed, including both verbal utterances and actions performed with the software. Transcript segments were coded on several dimensions, including content categories such as whether participants were discussing issues of evidence or using empirical or theoretical concepts. Essays were scored according to the strength and inferential difficulty of the evidential relations they cited. 
Although no significant differences were found on outcome measures related to the post-test and essays, there were definitive process differences. Results confirmed our prediction that notation significantly impacts learners' discussion of evidential relations. Analyses focused on the contents of participants' representations and their elaborations on (revisitations and reuse of) information and beliefs once they are represented. The results of these analyses indicated that visually structured and constrained representations provide guidance that is not afforded by plain text. Users of Matrix and Graph revisited previously discussed ideas more often than users of Text, as was predicted from the greater salience of ideas and prompting for missing relations in the more structured representations. However, not all guidance is equal, and more prompting is not necessarily better. Text and Matrix users represented more hypotheses and Matrix users represented far more evidential relations than were considered relevant by our own analysis of the problem. Matrix users revisited prior data and hypotheses mainly to fill in the matrix cells that relate them. They revisited relations far more often than Text or Graph users, but often appeared to be doing this because they were attempting to make relationships between weakly or equivocally related items due to the exhaustive prompting of the matrix. A representation such as Graph may guide students to consider evidence without making them unfocused.

We found no significant differences between the groups' post-test scores (recognition of factual information) and essay scores (using various measures of quality of inference), although all trends were in the predicted direction. These results were disappointing, but not surprising. Participants spent less than an hour on task, and this may not have been enough time for learning outcomes to develop fully. We did find that the contents of the Graph representations overlapped with the content of those participants' essays more than the corresponding representations overlapped in the Text or Matrix conditions. This result suggests that the work done using evidence maps had greater influence on participants' views of the problem as expressed in the essays.

\subsection{Appropriation of Representations for Online Collaboration}

All of the foregoing studies were undertaken with face-to-face collaboration of participants, yet online learning is becoming increasingly important, especially in higher education. We conducted a follow-up study designed to explore how the roles of representations in online learning might shift, with possible implications for the relevance of representational guidance (Suthers, Hundhausen, \& Girardeau, 2003). Although asynchronous learning environments are most prevalent, we chose to begin with a study of synchronous online collaboration so that the data would be comparable to our synchronous face-to-face data. This study was undertaken with a version of the Belvedere 3.0 research software that supported synchronous computermediated communication (CMC) with a textual "chat" provided in addition to the graph representation and information pages (Figure 3). 


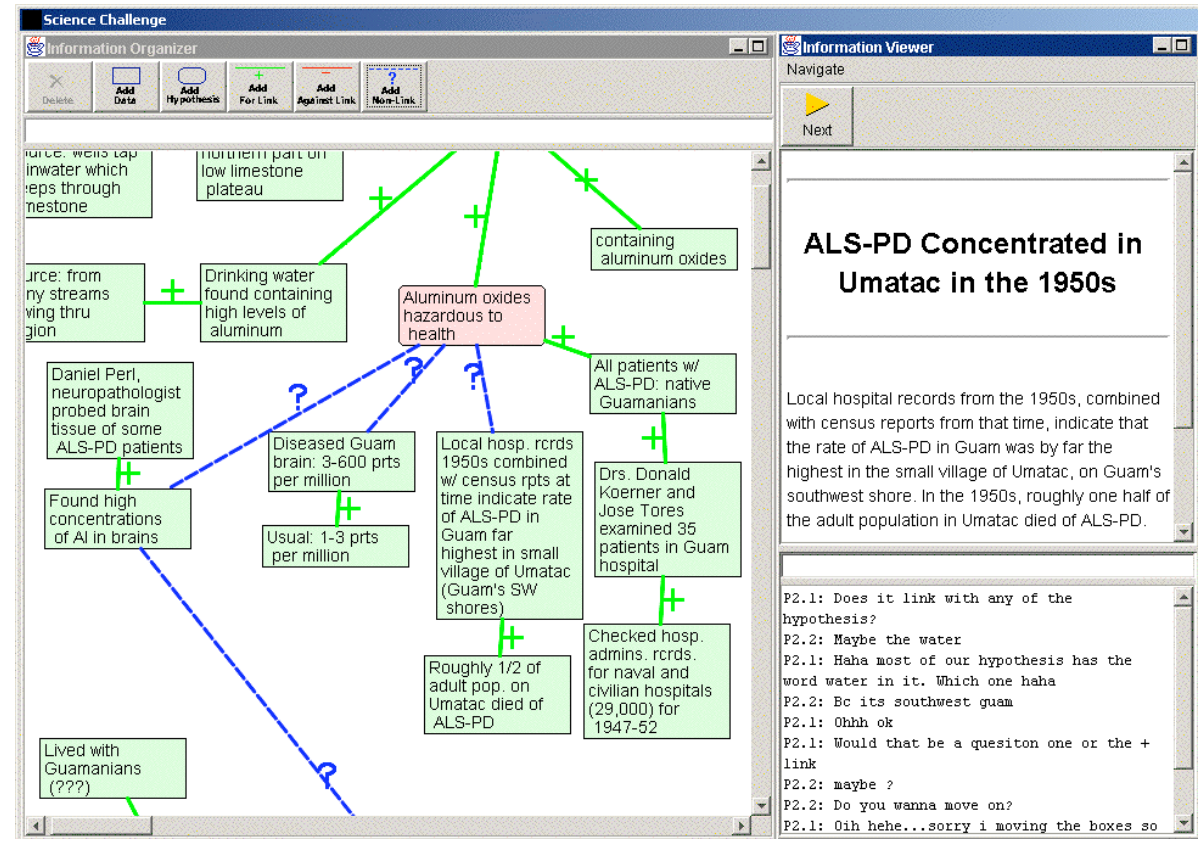

Figure 3. “Graph” software, synchronous CMC study

Extensive prior research has compared the performance of face-to-face collaborators with the performance of users of various forms of technology-mediated communication. Many of these studies show degradation of both problem-solving performance and interpersonal communication due to the reduced modes of interaction associated with technology-mediated communication (Doerry, 1996; Olson \& Olson, 2000). However, other studies show that people can compensate for and even benefit from restricted interaction (Burgoon et al., 2002; Herring, 1999), and that factors extrinsic to the technology itself may play a role (Walther, 1994). It was not our intent to replicate these results: our focus was on how the roles of external representations in supporting collaboration might change when going online, especially in ways that might affect the relevance of representational guidance. Two hypotheses were considered without prejudice:

(H1) Visual knowledge representations will play less of a role in guiding discourse online because without co-presence the representations do not as easily function to convey "taken as shared" information, and gestural references are more difficult online (Olson \& Olson, 2000).

(H2) Visual knowledge representations will play a greater role in supporting discourse online because participants will make use of them to make up for the reduced bandwidth of the chat tool as compared to speech. 
We conducted sessions with 10 pairs of students using the CMC version of Belvedere 3.0, and compared these sessions to the face-to-face graph sessions from the previous study in order to identify how the roles of representations in supporting collaboration might change. Other than the use of CMC, the protocols and measures were identical to the previous study.

Our quantitative results provided adequate evidence for the second hypothesis (Suthers, Hundhausen et al., 2003). In the online condition, a greater proportion of communicative acts relevant to the problem domain were undertaken in the graphical knowledge representation as opposed to spoken or chat communications. (Examples of communicative acts in the shared graphical medium include creating new data or hypothesis objects or linking two such objects together.) This was related to a shift in the role of the graph representation from object of discourse in the face-to-face condition to medium of discourse in the CMC condition. Online participants introduced new ideas directly in the graph medium (rather than in the chat) by modifying the representation far more often than face-to-face participants, who almost always introduced and discussed new ideas verbally before modifying the graph representation. As a consequence, in the online condition there was greater use of categories supported by the software (i.e., evidential relations and epistemic classifications). The chat was used primarily for social banter and task management (e.g., coordinating access to information pages and allocating responsibility for graph edits), and occasionally for problem-related discussion that was not supported by the graph representations (e.g., deciding how to interpret problematic information).

However, there was also qualitative evidence for the first hypothesis. Our informal review of the transcripts shows many examples of poorly coordinated activity in the online groups, such as disconnects between the activity in the workspace and the verbal activity in the chat. Also, we observed less use of gestural deixis ${ }^{4}$ and less rich discussion in the online condition. A subsequent analysis provided further evidence for H1 (Suthers, Girardeau, \& Hundhausen, 2003). In face-to-face collaboration, deixis was accomplished quite effectively through gesture. Gesture is spatially indexical: it can select any information in the shared visual space, regardless of when that information was previously encountered or introduced, making it an effective device for integrating old and new information. We did an analysis to determine what filled the functional role of gesture in the online environment. Online collaborators accomplished reference through verbal deixis and direct manipulation rather than gestural deixis. (See also Gergle, Kraut, \& Fussell, 2004.) As participants used it, verbal deixis in the chat tool was temporally indexical: it most often selected re-

${ }^{4}$ Deictic referencing, or deixis, is a reference to an entity in the extra-linguistic context. Deixis can be accomplished verbally with indexical terms such as "this," "it," and/or with gestures such as pointing or computer-aided highlighting. 
cently manipulated items (e.g., typing "what do you think?" after modifying the representation).

These results raised the question of whether and how online participants revisited prior information. Direct manipulation of the representations seemed to play this role most effectively, and indeed constituted an alternative means through which some aspects of communication about problem solution took place. However, communication in an evidence map is limited to propositions in the domain and the evidential relations between them. ${ }^{5}$ Direct manipulation is in a sense "first order." Higher order reflections such as discussion of possible interpretations of the information available are undertaken more often in the verbal media (speech or chat). Putting these observations together, there is a danger that online discourse may be less reflective, especially in its integration of new and prior information, because the more expressive and reflective mode of interaction-chat-focuses on recent (temporally indexed) items; while the easiest means of reintroducing prior information is through direct manipulation. This reasoning is consistent with our finding that online participants had lower scores on measures of information integration in their essays.

Having evidence for both hypotheses, we concluded that they are not in direct conflict, and may be synthesized as follows: Lack of mutual awareness of orientation towards shared representations may result in poorer coordination of immediate activity and the thinking behind it (H1). At the same time, greater reliance may be placed on those very representations as the medium through which activity takes place, biasing activity towards actions best supported by the representations (H2). From this work we learned that online discourse will not be confined to the medium provided for natural language interaction: it will be distributed across all mutable representations and influenced by the properties of those representations. Therefore, close attention must be paid to the design of affordances for argumentation in all representations provided to online collaborators. We also learned that the role of external representations as aids for integrating old and new information in an interactive, conversational manner could be weakened online due to the awkwardness of or lack of deictic affordances. Designers of online learning environments are advised to seek more natural means of referencing the contents of shared representations, particularly in conjunction with verbal communication. For example, chat or discussion tools might be designed to enable easy insertion of visual references to elements of other representations being discussed. Designers might also investigate other methods for helping online collaborators mutually attend to prior information, such as redisplay of prior information along with reflection prompts provided after a period of time.

${ }^{5}$ The phenomenon discussed here may be independent of what is represented. Other researchers have observed an initial resistance to formalization, even in representations that are intended to map discussion or argumentation rather than evidence. See for example (Shipman \& McCall, 1994). 


\subsection{Enhancing Knowledge Construction in Asynchronous Collaboration}

The most recent experimental study in this line of work was conducted in an asynchronous setting to inform this common form of online learning (Mayadas, 1997). This study focused on the question of whether conceptually explicit representations such as evidence maps can improve on the prevalent tool for online learning, namely threaded discussions. Although the lack of time-pressure in discussion forums may support more reflective contributions than synchronous communication (e.g., Hawkes \& Romiszowski, 2001), online interaction can also suffer from incoherence due to the violation of adjacency conventions for topic maintenance (Herring, 1999) and the coarse granularity of referencing (Reyes \& Tchounikine, 2003). Furthermore, there can be a lack of convergence due to the intrinsically divergent representations used in threaded discussion (Hewitt, 2001) and a bias towards addressing recently posted messages (Hewitt, 2003). The shared knowledge being constructed is not made explicit by typical CMC tools, and hence it is difficult to find relevant contributions, place one's own contribution in the relevant context, or quickly assess the outcome of the discussion (Suthers, 2001a; Turoff, Hiltz, Bieber, Fjermestad, \& Rana, 1999). Suthers (2001a) argued that if the conceptual development of the conversation can be made explicit and each contribution to the discussion can be referenced to a component of this conceptual representation, interactional coherence may improve because the conceptual relevance of each contribution is clear (see also van der Pol, Admiraal, \& Simons, 2006), and convergence may improve because multiple contributions referencing a given topic are collected together. We conducted an experimental test of these ideas in which two forms of conceptually-enhanced support were compared to each other and to a threaded discussion control condition (Suthers, Vatrapu, Medina, Joseph, \& Dwyer, 2007; Suthers, Vatrapu, Medina, Joseph, \& Dwyer, in press).

Based on reasons outlined at the beginning of this chapter, our primary hypothesis claimed:

(H1) Collaborative knowledge construction is more effectively supported by environments that make conceptual objects and relations explicit.

This primary hypothesis does not specify the relationship between knowledge representations and the conversation that accompanies the creation of those representations. Our secondary hypotheses are alternative elaborations of $\mathrm{H} 1$, arguing for either maintaining the distinction between discussion and knowledge representations or combining the two, as detailed next.

One could argue that discussion representations should be embedded in or mixed with the conceptual representations to contextualize the discussion and facilitate ease of reference (e.g., by simple attachment of notes to the objects to which they refer). A usability argument can also be made: it may be easier to manage a single workspace than interactions distributed across multiple tools. This reasoning led to the second hypothesis: 
(H2) Collaborative knowledge construction is more effectively supported if conversational and conceptual representations are tightly integrated.

The third hypothesis is motivated by the observation that conversational structures and conceptual structures are different: conversation relies on regularities in adjacency and focus shifts for coherence (Grosz \& Sidner, 1986; Sacks, Schegloff, \& Jefferson, 1974), while conceptualizations may be organized according to diverse ways of modeling or systematizing knowledge about the world. Therefore, separate tools will enable designers to optimize representations to meet the distinct structural needs of conversation and conceptualization in a given domain of discourse. Explicit referencing can be used to make the connection between the two representations (Mühlpfordt \& Wessner, 2005; Suthers, 2001a). This reasoning leads us to the third hypothesis, which is in opposition to the second:

(H3) Collaborative knowledge construction is more effectively supported if the distinction between discussion and conceptual models is reflected in the representations provided.

We constructed three software environments in order to test these hypotheses. All three of the environments had an information browser on the upper left side in which materials relevant to the task were displayed, and a shared on the right hand side in which participants could share information they gather from the problem materials as well as their own interpretations and other ideas (Figure 4). Changes made to the workspace by each participant were propagated to other participant's displays under an asynchronous update protocol to simulate asynchronous interaction common in online learning. An action taken by one participant did not appear in the other participant's workspace until after the receiving participant "took a break" by playing a game of Tetris ${ }^{\mathrm{TM}}$.

The three environments differed on the nature of the shared workspace. The shared workspace in the Text condition was a conventional threaded discussion tool. This is the control condition for testing the above hypotheses, since the workspace only provided explicit support for representation of discussion structure (subject headings and reply relations). Motivated by $\mathrm{H} 2$, the shared workspace for the Graph condition was based on the same Belvedere-derived evidence map representation as the previous studies with the addition of an embedded note object that supported a simple linear (unthreaded) discussion that was interactionally asynchronous and could be linked in the evidence map like any other object. Motivated by H3, the shared workspace of the Mixed condition (Figure 4) included both a threaded discussion tool (lower left) and an evidence-mapping tool for representing conceptual structure in the same manner as the Graph condition, except that there were no embedded notes in the Mixed version of the evidence map. Instead, one could embed references to evidence map objects in the threaded discussion messages by clicking on the relevant graph object while composing the message. The references showed up as small icons in the message that could be clicked on to highlight the corresponding object in the evidence map (as exemplified in Figure 4). 


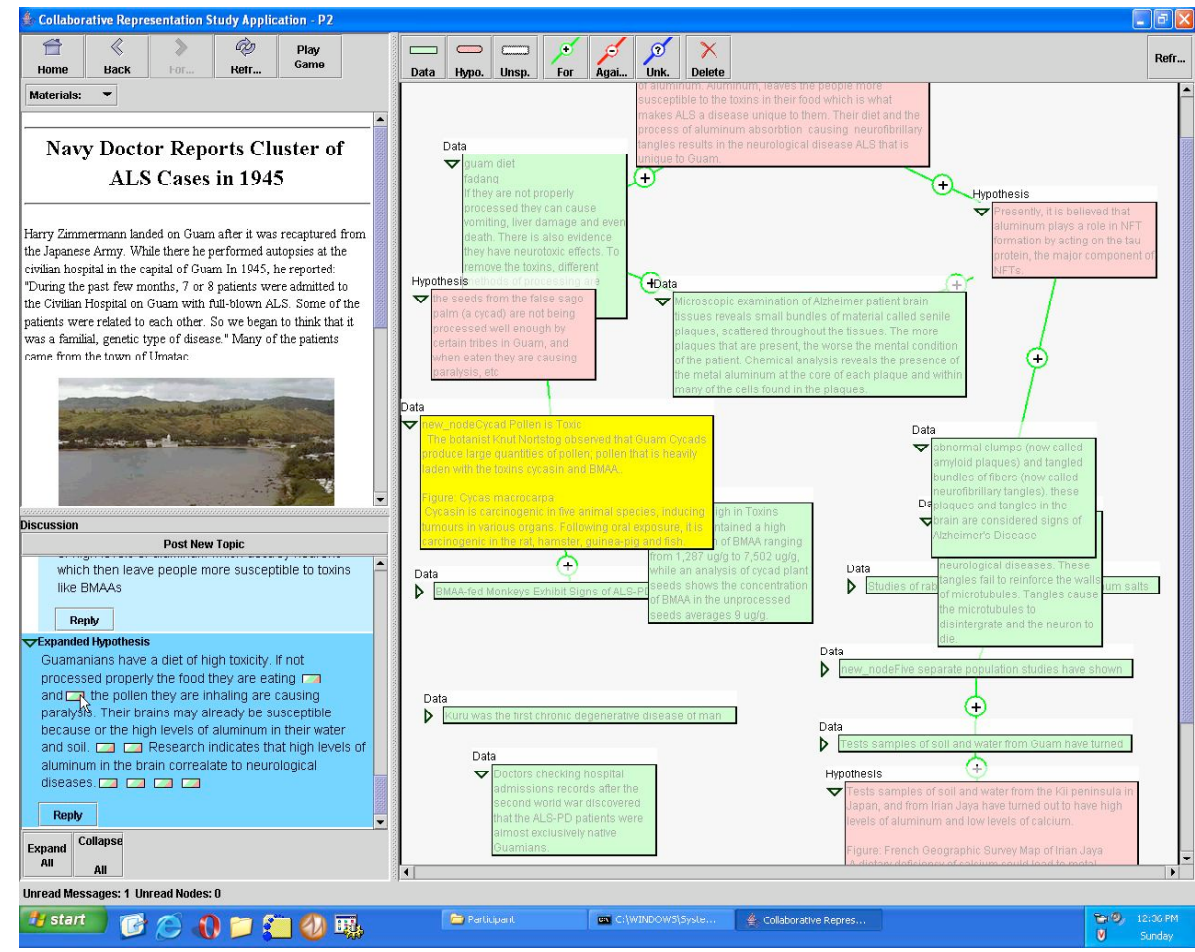

Figure 4. "Mixed" software from the asynchronous CMC study

Materials were prepared based on the professional literature concerning a complex public health problem: a disease that historically occurred in the native population on the island of Guam. The materials suggested several distinct possible causes of the disease, and provided mixed evidence for and against each cause. Relevant evidence was distributed in a hidden profile such that if participants did not share any information each participant would have evidence favoring a suboptimal disease hypothesis. Sharing was required to reject these hypotheses and construct a more complex explanation. In each dyad, Participant 1 (P1) received evidence for aluminum in the water and against genetic causes; Participant 2 (P2) received evidence against aluminum and for genetic causes; and both participants received evidence for and against cycad seeds as the source of a neurotoxin as well as crucial information about native diets that, when brought together, points to seed-eating bats as the vector by which 
this toxin gets into humans. The articles included distracter information as well as relevant evidence. ${ }^{6}$

Participants were directed to use the computer workspace to share information with their partner, and were told that this was necessary to identify the correct cause of the disease and to perform well on the essay and post-test to be given at the end. At the conclusion of their problem solving, each individual was asked to write an essay detailing the disease hypotheses considered and the evidence for and against those hypotheses, and to identify the best explanation for the disease. One week after their session, participants were directed to take an online post-test. This test included questions that tested participants' memory for distracter information, memory for relevant information, and facts that required integration of multiple items of relevant information. "High integration" questions required integration of information that occurred far apart in the materials (in Suthers \& Hundhausen, 2003's terms, there is a large "inferential span"). The questions were based on information given uniquely to one or the other participant, enabling us to assess the residue of information sharing.

Our analyses addressed outcomes, based on content analyses of the essays and scoring of the post-test; and session processes, based on quantitative analyses of elaboration on hypotheses. Two lines of evidence support H1, based on process and outcome data, as detailed below.

The process data shows clearly that there was more elaboration on hypotheses in both of the environments that made conceptual objects and relations explicit (Graph and Mixed) as compared to the environment that did not (Text). Hypotheses were stated earlier in the experimental session and there was more elaboration on the hypotheses individually as well as collectively. Furthermore, Graph users considered more hypotheses. These results are consistent with the representational guidance effects demonstrated for face-to-face interaction in the classroom study (Toth et al., 2002) and the laboratory study (Suthers \& Hundhausen, 2003) discussed previously in this chapter. In summary, process measures suggest that more knowledge construction takes place when interaction is supported by conceptual representations.

Turning to outcomes, the treatment conditions did not differ in optimality of conclusion in the essays: relatively few participants in all conditions identified the bats-as-vector explanation for how the cycad toxin gets into humans. However, pairs in the Graph condition were more likely to express the same (not necessarily optimal) conclusions in their essays. This convergence cannot be attributed to a paucity of alternatives: the process data showed that Graph users considered more hypotheses than the others, which makes their convergence even more notable. The convergence is not due to more effective information sharing per se: there were no differences on whether information given to one participant appeared in the other's essay, or on memory for information given to one's partner (from the post-test analysis). Also, a later analysis showed that Text users actually shared more information during the session (Suthers, Medina, Vatrapu, \& Dwyer, 2007). (There

${ }^{6}$ Archived at http://lilt.ics.hawaii.edu/lilt/papers/2006/Suthers-et-al-CE-2006/. 
session (Suthers, Medina, Vatrapu, \& Dwyer, 2007). (There was a greater tendency of the Text participants to simply cut and paste entire articles into their text messages and leave discussion for the end.) Technologies that enable people to share more information do not necessarily lead to effective use of that information (Dennis, 1996). Given the process data just reviewed, it is plausible that something beyond information sharing, such as collaborative consideration of hypotheses during the study sessions had an effect on convergence of the participants' conclusions.

On the other hand, the lack of differences on quality of solution may be counted as evidence against H1. Also, the failure of the Mixed condition in some analyses to display the advantages claimed by $\mathrm{H} 1$ may also be considered as evidence against $\mathrm{H} 1$, but the dual workspace is a confounding factor, as it requires managing two representations (Ainsworth, Bibby, \& Wood, 1998). Participants in the Mixed condition may have converged the least because the dual workspaces provide more variation in strategies for using the workspaces, increasing the possibility that members of a pair will look at different material.

Turning to the comparison between $\mathrm{H} 2$ (in favor of integrated representations such as Graph) and H3 (in favor of distinct discussion and conceptual representations such as Mixed), significant differences on direct comparisons between Graph and Mixed are limited to the result that Graph users scored higher than Mixed users on post-test questions requiring integration of information that was distributed across the materials. The distribution of information across two media in Mixed may have posed a barrier to integration of that information, obscuring the advantage of Mixed's evidence map. However, there is indirect evidence bearing on the choice between $\mathrm{H} 2$ and H3. All other statistical analyses in which there was a significant advantage for one of the conditions over the others included an advantage of Graph over Text. In contrast, Mixed was sometimes advantageous to Text, sometimes not, but never was advantageous to Graph, and sometimes yielded the worst results. Since Graph and Matrix were introduced as competing alternatives to threaded discussions, support for H2 (Graph) is stronger than for H3 (Mixed).

The primary conclusion of this study - that collaborative knowledge construction is fostered by conceptual representations - not only adds to the growing literature on representational guidance for collaborative learning, but also has practical implications. Should threaded discussion tools be replaced with knowledge mapping tools in online learning? Although that is the direction in which the results point, it would be a brash conclusion to draw from this experiment alone, as it is limited in many ways. We studied dyads interacting over a relatively short period of two hours. Dozens of students interacting over the course of a semester (even if divided into smaller groups as is generally recommended in ALN implementations) would generate much more complex artifacts. Any workspace has a limited useful life before it becomes important to "rise above" the clutter and start fresh (Scardamalia, 2004). The subject matter, task structure, and nature of the representations used could also affect results. However, in conjunction with previous work the present results merit extending the research program beyond the laboratory by undertaking action research in which richer interactive representations are studied in settings of educational practice. 


\section{Related Work}

During this time, other researchers have undertaken related studies of representational effects using conceptually explicit representations. For example, Veerman (2003) compared Allaire Forums (asynchronous online discussion), Belvedere 2.0 (using synchronous discussion with a chat tool) and NetMeeting (internet videoconferencing) in a heterogeneous design (the activities were not identical). Among other differences, Veerman observed a greater percentage of argumentation related content, particularly counter-arguments, in Belvedere, a result that seems consistent with the Toth et al. (2002) result on discrepant evidence. Schwartz, Neuman, Gil, \& Ilya (2002) showed that argument maps were superior to pro-con tables in supporting students' collaborative argumentation and essay writing, but these differences were not internalized individually during the relatively short study. Others have studied alternative instructional strategies for using conceptually explicit representations in collaborative learning (e.g., Lund, Molinari, Séjourné, \& Baker, 2007; Stoyanova \& Kommers, 2002). Related work may be found in (Andriessen, Baker, \& Suthers, 2003)

The studies reported above were conducted using experimental manipulations and quantitative analyses. This methodology is valuable for hypothesis testing, but is weaker for discovery of the actual practices by which participants make use of resources to accomplish their goals. Coding and statistical aggregation obscures what participants are doing as they try to make sense of the problem and the situation at multiple levels. For these reasons, (Suthers, 2006b), following (Koschmann et al., 2005; Stahl, 2006), argued for a turn towards the study of practices of individual and intersubjective meaning-making through which learning is ultimately accomplished, and suggested that sequential analyses of interaction are more appropriate for understanding how the cognitive and social affordances of technologies such as knowledge maps are appropriated by participants as well as influencing their interaction. Pursuing this agenda, the author re-examined the data from the synchronous laboratory study using the concept of uptake as the fundamental unit of analysis (Suthers, 2006a). Subsequently, we have explicated a formal and theoretically motivated basis for such analysis (Suthers, Dwyer, Medina, \& Vatrapu, 2007). Early results from associated studies include an apparent pattern of successful collaboration in which information sharing is followed by subsequent "round trips" of negotiation of agreement, and the observation that while information sharing takes place in the knowledge map, parallel linguistic channels are used for these subsequent negotiations. Other recent analyses of meaning-making with conceptually explicit representations include (Mirza, Tartas, Perret-Clermont, \& de Pietro, 2007; Schwarz \& De Groot, 2007).

\section{Conclusions}

The studies of representational guidance for collaborative learning summarized in this chapter were motivated by the idea that some roles of representations in supporting learning are endemic to collaborative situations and that logical and perceptual 
differences between representations may influence how they fill these roles. A laboratory study confirmed several predicted process differences, including discussion of evidence and revisitation of prior information, as well as suggestive results indicating that the work done with graphs had greatest impact on participants' understanding of the problem. A study of the products of students' classroom work showed similar effects of representation on consideration of discrepant evidence, this effect being amplified by a coordinated set of peer-evaluation rubrics calling for evaluation of discrepant evidence. The online study showed that all actionable/mutable representations will be appropriated as part of the discourse medium (not just the intended discussion tools), and therefore we may expect representational guidance to be enhanced in online discourse. This work was continued in a study of asynchronous interaction, which confirmed the influences of conceptually explicit representations on collaborative processes, leading to greater integration of information by individuals and greater convergence of conclusions by pairs (even after they considered a diversity of alternatives).

The immediate implication of this work is that system designers should treat representational design as design of resources for conversation between learners. A designer or teacher might ask: What activities does a given representational notation suggest or prompt for? Do the actions that can be performed on a shared representation in this notation correspond to the potential ideas that we want learners to negotiate and distinctions we want them to attend to? Do the resulting representations express and make salient the ideas and relationships that learners should revisit and relate to new information? Are the needs that should be addressed by subsequent activity, such the lack of information, made obvious? Do the representations capture important aspects of learners' thinking and expose conflicts between alternative solutions or perspectives? Stepping beyond the scope of the studies reported here, one might ask: does the notation provide the preferred vocabularies and representational perspectives that constitute both the target skill to be learned as an aspiring member of a community, and focus learning activity on ways of approaching a problem that are productive? Representational notations are not determinants of behavior, but when the features of representations are coordinated with the design of other elements of a learning situation they can guide behavior. Activity theory (Cole \& Engeström, 1993; Wertsch, 1998) tells us that tools and artifacts (among other things) mediate the influences of various learning resources on the learner, such as other individuals, community norms and roles. Therefore, the impact of the representational choices we make in designing these tools is not limited merely to the direct effects of representations. The impact of these choices will be amplified to the extent that the representations mediate how other resources in the human-computer system bear upon the learning activity. 


\section{Acknowledgements}

This work was supported by the National Science Foundation grants \#9873516 (Knowledge and Distributed Intelligence) and \#0093505 (CAREER), and was conducted in collaboration with numerous individuals acknowledged in the author's cited publications.

\section{References}

Ainsworth, S. E., Bibby, P. A., \& Wood, D. J. (1998). Analyzing the costs and benefits of multi-representational learning environments. In M. W. van Someren, P. Reimann, H. P. A. Boshuizen \& T. de Jong (Eds.), Learning with Multiple Representations (pp. 120-134). Amsterdam: Elsevier Science, Ltd.

Andriessen, J., Baker, M., \& Suthers, D. D. (2003). Arguing toLearn: Confronting Cognitions in Computer-Supported Collaborative Learning Environments. Dordrecht: Kluwer.

Bell, P. (1997). Using argument representations to make thinking visible for individuals and groups. In Proc. 2nd International Conference on Computer Supported Collaborative Learning (CSCL'97) (pp. 10-19). Toronto University of Toronto.

Brna, P., Cox, R., \& Good, J. (2001). Learning to think and communicate with diagrams: 14 questions to consider. Artificial Intelligence Review, 15(1-2), 115-134.

Burgoon, J. K., Bonito, J. A., Ramirez Jr., A., Dunbar, N. E., Kam, K., \& Fischer, J. (2002). Testing the interactivity principle: Effects of mediation, propinquity, and verbal and nonverbal modalities in interpersonal interaction. Journal of Communication, 657-677.

Cavalli-Sforza, V., Weiner, A. W., \& Lesgold, A. M. (1994). Software support for students engaging in scientific activity and scientific controversy. Science Education, 78(6), 577599.

Cole, M., \& Engeström, Y. (1993). A cultural-historical approach to distributed cognition. In G. Salomon (Ed.), Distributed Cognitions: Psychological and Educational Considerations (pp. 1-46). Cambridge: Cambridge University Press.

Dennis, A. R. (1996). Information exchange and use in group decision making: You can lead a group to information, but you can't make it think. MIS Quarterly, 20(4), 433-457.

Doerry, E. (1996). An Empirical Comparison of Copresent and Technologically-Mediated Interaction based on Communicative Breakdown. University of Oregon.

Erickson, T., \& Kellogg, W. A. (2000). Social translucence: An approach to cesigning systems that support social processes. ACM Transactions on Computer-Human Interaction, 7(1), 59-83.

Gergle, D., Kraut, R. E., \& Fussell, S. R. (2004). Action as language in a shared visual space. In Proceedings of the 2004 ACM Conference on Computer Supported Cooperative Work (pp. 487-496). Chicago, Illinois: ACM Press.

Grosz, B. J., \& Sidner, C. L. (1986). Attention, intentions, and the structure of discourse. Computational Linguistics, 12(3), 175-204.

Guzdial, M., Hmelo, C., Hubscher, R., Newstetter, W., Puntambekar, S., Shabo, A., et al. (1997). Integrating and guiding collaboration: Lessons learned in computer-supported collaboration learning research at Georgia Tech. In Computer-Supported Collaborative Learning (pp. 91-100). Toronto, Ontario.

Hawkes, M., \& Romiszowski, A. (2001). Examining the reflective outcomes of asynchronous computer-mediated communication on inservice teacher development. Journal of Technology and Teacher Education, 9(2), 285-308. 
Herring, S. C. (1999). Interactional coherence in CMC. Journal of Computer Mediated Communication, 4(4).

Hewitt, J. (2001). Beyond threaded discourse. International Journal of Educational Telecommunications, 7(3), 207-221.

Hewitt, J. (2003). How habitual online practices affect the development of asynchronous discussion threads. Journal of Educational Computing Research, 28(1), 31-45.

Koschmann, T., Zemel, A., Conlee-Stevens, M., Young, N., Robbs, J., \& Barnhart, A. (2005). How do people learn: Member's methods and communicative mediation. In R. Bromme, F. W. Hesse \& H. Spada (Eds.), Barriers and Biases in Computer-Mediated Knowledge Communication (and how they may be overcome) (pp. 265-294). Amsterdam: Kluwer Academic Press.

Kotovsky, K., \& Simon, H. A. (1990). What makes some problems really hard: Explorations in the problem space of difficulty. Cognitive Psychology, 22, 143-183.

Kreijns, K., \& Kirschner, P. A. (2004). Designing sociable CSCL environments. In J.-W. Strijbos, P. A. Kirschner \& R. L. Martens (Eds.), What We Know About CSCL And Implementing It In Higher Education (pp. 221-243): Springer.

Larkin, J. H., \& Simon, H. A. (1987). Why a diagram is (sometimes) worth ten thousand words. Cognitive Science, 11, 65-99.

Lohse, G. L. (1997). Models of graphical perception. In M. Helander, T. K. Landauer \& P. Prabhu (Eds.), Handbook of Human-Computer Interaction (pp. 107-135). Amsterdam: Elsevier Science B.V.

Lund, K., Molinari, G., Séjourné, A., \& Baker, M. (2007). How do argumentation diagrams compare when student pairs use them as a means for debate or as a tool for representing debate? International Journal of Computer Supported Collaborative Learning, 2(2).

Mayadas, F. (1997). Asynchronous learning networks: A Sloan Foundation perspective. Journal of Asynchronous Learning Networks, 1 , http://www.aln.org/alnweb/journal/jaln issue1.htm\#mayadas.

Mirza, N. M., Tartas, V., Perret-Clermont, A.-N., \& de Pietro, J.-F. (2007). Using graphical tools in a phased activity for enhancing dialogical skills: An example with Digalo. International Journal of Computer Supported Collaborative Learning, 2(2).

Mühlpfordt, M., \& Wessner, M. (2005). Explicit referencing In chat supports collaborative learning. In T. Koschmann, D. D. Suthers \& T.-W. Chan (Eds.), Computer Supported Collaborative Learning: The Next 10 Years! (pp. 460-469). Mahwah, NJ: Lawrence Erlbaum Associates.

Novick, L. R., \& Hmelo, C. E. (1994). Transferring symbolic representations across nonisomorphic problems. Journal of Experimental Psychology: Learning, Memory and Cognition, 20(6), 1296-1321.

O'Neill, D. K., \& Gomez, L. M. (1994). The collaboratory notebook: A distributed knowledge-building environment for project-enhanced learning. In Proceedings of Ed-Media '94. Charlottesville, VA AACE.

Olson, G. M., \& Olson, J. S. (2000). Distance matters. Human-Computer Interaction, 15(2/3).

Paolucci, M., Suthers, D. D., \& Weiner, A. (1996). Automated advice-giving strategies for scientific inquiry. In C. Frasson, G. Gauthier \& A. Lesgold (Eds.), Third International Conference on Intelligent Tutoring Systems (ITS'96) (1086 ed., pp. 372-381). Monteal: Springer.

Puntambekar, S., Nagel, K., Hübscher, R., Guzdial, M., \& Kolodner, J. (1997). Intra-group and intergroup: An exploration of learning with complementary collaboration tools. In Proc. 2nd International Conference on Computer Supported Collaborative Learning (CSCL'97) (pp. 207-214). Toronto: University of Toronto. 
Ranney, M., Schank, P., \& Diehl, C. (1995). Competence versus performance in critical reasoning: Reducing the gap by using Convince Me. Psychological Teaching Review, 4(2), 151-164.

Reyes, P., \& Tchounikine, P. (2003). Supporting emergence of threaded learning conversations through augmenting interactional and sequential coherence. In B. Wasson, S. Ludvigsen \& U. Hoppe (Eds.), Designing for Change in Networked Learning EnvironmentsProceedings of Conference CSCL 2003 (pp. 83-92). Dordrecht: Kluwer Academic Publishers.

Roschelle, J. (1996). Designing for cognitive communication: Epistemic fidelity or mediating collaborating inquiry. In D. L. Day \& D. K. Kovacs (Eds.), Computers, Communication \& Mental Models (pp. 13-25). London: Taylor \& Francis.

Sacks, H., Schegloff, E. A., \& Jefferson, G. (1974). A simplest systematics for the organization of turn-taking for conversation. Language, 50(4), 696-735.

Scardamalia, M. (2004). CSILE/Knowledge Forum ${ }^{\circledR}$. In Education and technology: An encyclopedia. (pp. 183-193). Santa Barbara: ABC-CLIO.

Scardamalia, M., Bereiter, C., Brett, C., Burtis, P. J., Calhoun, C., \& Smith Lea, N. (1992). Eductional applications of a networked communal database. Interactive Learning Environments, 2(1), 45-71.

Schwarz, B., \& De Groot, R. (2007). Argumentation in a changing world. International Journal of Computer Supported Collaborative Learning, 2(2).

Schwarz, B., Neuman, Y., Gil, Y., \& Ilya, M. (2002). Construction of collective and individual knowledge in argumentative activities: An experimental study. Journal of the Learning Sciences, 12(2).

Shipman, F. M., III, \& McCall, R. (1994). Supporting knowledge-base evolution with incremental formalization, CHI94 (pp. 285-291). Boston, MA: ACM Press.

Smolensky, P., Fox, B., King, R., \& Lewis, C. (1987). Computer-aided reasoned discourse, or, how to argue with a computer. In R. Guindon (Ed.), Cognitive Science and its Implications for Human-Computer Interaction: Lawrence Erlbaum.

Stahl, G. (2006). Group Cognition: Computer Support for Collaborative Knowledge Building. Cambridge, MA: MIT Press.

Stenning, K., \& Oberlander, J. (1995). A cognitive theory of graphical and linguistic reasoning: logic and implementation. Cognitive Science, 19, 97-140.

Stoyanova, N., \& Kommers, P. (2002). Concept mapping as a medium of shared cognition in computer-supported collaborative problem solving. Journal of Interactive Learning Research, 13(5), 111-134.

Suthers, D. D. (2001a). Collaborative representations: Supporting gace to face and online knowledge-building discourse. In Proceedings of the 34th Hawai i International Conference on the System Sciences (HICSS-34), January 3-6, 2001, Maui, Hawai i (CD-ROM): Institute of Electrical and Electronics Engineers, Inc. (IEEE).

Suthers, D. D. (2001b). Towards a systematic study of representational guidance for collaborative learning discourse. Journal of Universal Computer Science, 7(3).

Suthers, D. D. (2006a). A qualitative analysis of collaborative knowledge construction through shared representations Research and Practice in Technology Enhanced Learning, 1(2), 128.

Suthers, D. D. (2006b). Technology affordances for intersubjective meaning-making: A research agenda for CSCL. International Journal of Computers Supported Collaborative Learning, 1(3), 315-337.

Suthers, D. D., Connelly, J., Lesgold, A. M., Paolucci, M., Toth, E. E., Toth, J., et al. (2001). Representational and advisory guidance for students learning scientific inquiry. In K. D. F. a. P. J. Feltovich (Ed.), Smart Machines in Education: the Coming Revolution in Educational Technology (pp. 7-35). Cambridge, Massachusetts: AAAI Press, The MIT Press. 
Suthers, D. D., Dwyer, N., Medina, R., \& Vatrapu, R. (2007). A framework for eclectic analysis of collaborative interaction. In C. Chinn, G. Erkens \& S. Puntambekar (Eds.), The Computer Supported Collaborative Learning (CSCL) Conference 2007 (pp. 694-703). New Brunswick: International Society of the Learning Sciences.

Suthers, D. D., Girardeau, L. E., \& Hundhausen, C. D. (2003). Deictic roles of external representations in face-to-face and online collaboration. In B. Wasson, S. Ludvigsen \& U. Hoppe (Eds.), International Conference on Computer Support for Collaborative Learning 2003 (pp. 173-182). Dordrecht: Kluwer Academic Publishers.

Suthers, D. D., \& Hundhausen, C. (2003). An experimental study of the effects of representational guidance on collaborative learning. Journal of the Learning Sciences, 12(2), 183219.

Suthers, D. D., Hundhausen, C. D., \& Girardeau, L. E. (2003). Comparing the roles of representations in face-to-face and online computer supported collaborative learning. Computers \& Education, 41, 335-351.

Suthers, D. D., Medina, R., Vatrapu, R., \& Dwyer, N. (2007). Information sharing is incongruous with collaborative convergence: The case for interaction. In C. Chinn, G. Erkens \& S. Puntambekar (Eds.), The Computer Supported Collaborative Learning (CSCL) Conference 2007 (pp. 714-716). New Brunswick: International Society of the Learning Sciences.

Suthers, D. D., Toth, E. E., \& Weiner, A. (1997). An integrated approach to implementing collaborative inquiry in the classroom. In R. Hall, N. Miyake \& N. Enyedy (Eds.), Proceedings of the Second International Conference on Computer Support for Collaborative Learning (pp. 272-279). Toronto.

Suthers, D. D., Vatrapu, R., Medina, R., Joseph, S., \& Dwyer, N. (2007). Conceptual representations enhance knowledge construction in asynchronous collaboration. In C. Chinn, G. Erkens \& S. Puntambekar (Eds.), The Computer Supported Collaborative Learning (CSCL) Conference 2007 (pp. 704-713). New Brunswick: International Society of the Learning Sciences.

Suthers, D. D., Vatrapu, R., Medina, R., Joseph, S., \& Dwyer, N. (in press). Beyond threaded discussion: Representational guidance in asynchronous collaborative learning environments. Computers \& Education, doi:10.1016/j.compedu.2006.10.007.

Suthers, D. D., \& Weiner, A. (1995, October 17-20). Groupware for developing critical discussion skills. Paper presented at the First International Conference on Computer Support for Cooperative Learning, Bloomington, IN.

Suthers, D. D., Weiner, A., Connelly, J., \& Paolucci, M. (1995). Belvedere: Engaging students in critical discussion of science and public policy issues. In J. Greer (Ed.), Proceedings of AI-ED 95 - World Conference on Artificial Intelligence in Education (pp. 266-273). Washington, D.C.: AACE.

Tabak, I. (2004). Synergy: A complement to emerging patterns of distributed scaffolding. Journal of the Learning Sciences, 13(3), 205-335.

Toth, E. E., Suthers, D. D., \& Lesgold, A. M. (2002). "Mapping to know": The effects of representational guidance and reflective assessment on scientific inquiry. Science Education, 86(2), 264-286.

Turoff, M., Hiltz, S. R., Bieber, M., Fjermestad, J., \& Rana, A. (1999). Collaborative discourse structures in computer mediated group communications. Journal of ComputerMediated Communication, 4(4), Online: http://jcmc.huji.ac.il/

van der Pol, J., Admiraal, W., \& Simons, P. R. J. (2006). The affordance of anchored discussion for the collaborative processing of academic texts. Comptuer-Supported Collaborative Learning, 1(3), 339-357. 
Veerman, A. (2003). Constructive discussions through electronic dialogue. In J. Andriessen, M. Baker \& D. D. Suthers (Eds.), Arguing to Learn: Confronting Cognitions in ComputerSupported Collaborative Learning Environments (pp. 117-143). Dordrecht: Kluwer.

Walther, J. B. (1994). Anticipated ongoing interaction versus channel effects on relational communication in computer-mediated interaction. Human Communication Research, 20(4), 473-501.

Wertsch, J. V. (1998). Mind as Action. New York: Oxford University Press.

Zhang, J. (1997). The nature of external representations in problem solving. Cognitive Science, 21(2), 179-217. 